\title{
LES OF OPEN-CHANNEL FLOW WITH FREE-SURFACE FLUCTUATION
}

\author{
Akihiko NAKAYAMA ${ }^{1}$ and Satoshi YOKOJIMA ${ }^{2}$ \\ ${ }^{1}$ Member of JSCE, Ph.D., Professor, Grad. School of Science and Tech., Kobe University \\ (1-1, Rokkodai, Nada, Kobe 657-8501, Japan) \\ ${ }^{2}$ Member of JSCE, JSPS Research Fellow (DC), Grad. School of Science and Tech., Kobe University
}

\begin{abstract}
Large-eddy simulation of fully-developed turbulent open-channel flow has been conducted allowing motion of the free surface. The shape of the free surface, and hence flow domain, is filtered along with the flow field itself. Filtering of the free surface position introduces extra subgridscale (SGS) terms. Two different models for these new SGS terms are proposed and incorporated in actual LES calculations. Calculations with and without the free-surface SGS terms indicate that the effects of these terms may not be overwhelmingly large in subcritical flows, but they make some differences in the mean-velocity profile near the free surface and in the Reynolds stresses. The effects can be much more in spatially or temporally varying flows at higher Reynolds and Froude numbers.
\end{abstract}

Key Words : large-eddy simulation, open-channel flow, free-surface effects, subgrid-scale fluctuations

\section{INTRODUCTION}

Large-eddy simulation (LES) is now considered to be one of the most promising methods of turbulent-flow calculation in various applications including hydraulic engineering. Like other methods of turbulent-flow analysis, it has been better verified for internal flows with fixed boundaries. Flows encountered in hydraulic engineering often involve the free surface and its representation and effects on the rest of the flow must be taken into account when methods developed for internal flows are applied. Largeeddy simulations of open-channel flows have been conducted by several workers including Thomas et al. ${ }^{1)}$, Kawamura ${ }^{2)}$, Hodges \& Street $^{3)}$, Dimas \& Fialkowski ${ }^{4}$, Shen \& Yue ${ }^{5)}$, Kawahara ${ }^{6)}$ and Onitsuka \& $\mathrm{Nezu}^{7}$ ). Each of these authors treats the free surface differently with varying degrees of emphasis placed on its importance. Kawamura ${ }^{2)}$, Kawahara $^{(6)}$ and Onitsuka \& Nezu ${ }^{7)}$ assume no deformation of the free surface and apply the rigid-slip surface condition so that the methods for internal flows are applied with minor changes. Shen \& Yue ${ }^{5)}$ assumed small surface fluctuations and applied the linearized boundary conditions. Thomas et al. ${ }^{1)}$ considered larger fluctuation and used the Volume of Fluid (VOF) method on rectangular grid to track the moving boundary. Hodges \& Street $^{3)}$ also allowed a large surface deformation but used the boundary-conforming general coordinates. Thomas et al. ${ }^{1}{ }^{1}$ ignored the filtering effects of the deforming free surface, but Hodges \& Street $^{3)}$, Dimas \& Fialkowski ${ }^{4}$ ) and Shen \& Yue ${ }^{5)}$ recognize that when the free surface is allowed to move, filtering of the freesurface shape and the boundary conditions introduces the additional subgrid-scale (SGS) terms that need to be modeled. They have not, however, made detailed evaluation of the role of the free-surface SGS terms.

The main purpose of the present paper is to present the results of LES calculation of fully-developed open-channel flow considering the movement of the free surface and the effects of its filtering. Only subcritical Froude numbers are considered so that the amplitude of the free surface fluctuations are small. The present authors $^{8)}$ have conducted the direct numerical simulation (DNS) of such flows and the LES results will be evaluated by comparing with these data. From the DNS calculation assuming that the deviation of the instantaneous and local posi- 
tion of the free surface is small compared with the average depth, it has been found that a continuous function of horizontal position to represent the local and instantaneous position of the free surface is adequate, and the boundary condition may be linearized to be applied at the mean position. In this method of small-amplitude wave approximation, the filtering effects of the freesurface shape appears in the kinematic boundary condition as extra flux-like correlation terms. Details of modeling of this equation and the modeling of the extra SGS correlation terms are discussed in a separate paper ${ }^{9)}$. Here actual calculations are performed with and without these terms and their effects and importance are studied. Other aspects of the LES are kept close to standard as much as possible so that the effects of the free-surface motion and filtering are isolated and studied. The other issues of the freesurface effects such as those on the damping and anisotropy effects of the SGS stresses are not addressed.

\section{LES METHOD}

\section{(1) Governing equations}

We consider a fully-developed turbulent flow of mean depth $H$ in a two-dimensional open channel shown in Fig. 1, with $x_{1}$ the streamwise direction along the channel bed which is inclined by angle $\theta$ from the horizontal direction, $x_{2}$ the direction normal to the bed and positive upward and $x_{3}$ the transverse direction. The governing equations for the filtered velocity components $\left\langle u_{i}\right\rangle$ are

$$
\begin{gathered}
\frac{\partial\left\langle u_{i}\right\rangle}{\partial x_{i}}=0 \\
\frac{D\left\langle u_{i}\right\rangle}{D t}=-\frac{\partial\langle P\rangle}{\partial x_{i}}+g_{1} \delta_{1 i}+\frac{\partial}{\partial x_{j}}\left(-\tau_{i j}+2 \nu\left\langle S_{i j}\right\rangle\right)
\end{gathered}
$$

where $g_{1}=g \sin \theta, g_{2}=-g \cos \theta$ and $g_{3}=0$ are the components of the gravitational acceleration in the $x_{1}, x_{2}$ and $x_{3}$ directions, respectively,

$$
\begin{gathered}
\langle P\rangle=\langle p\rangle+g_{2}\left(H-x_{2}\right), \\
\left\langle S_{i j}\right\rangle=\frac{1}{2}\left(\frac{\partial\left\langle u_{i}\right\rangle}{\partial x_{j}}+\frac{\partial\left\langle u_{j}\right\rangle}{\partial x_{i}}\right)
\end{gathered}
$$

is the rate of deformation of the filtered flow, and

$$
\tau_{i j}=\left\langle u_{i} u_{j}\right\rangle-\left\langle u_{i}\right\rangle\left\langle u_{j}\right\rangle
$$

is the conventional SGS stress.

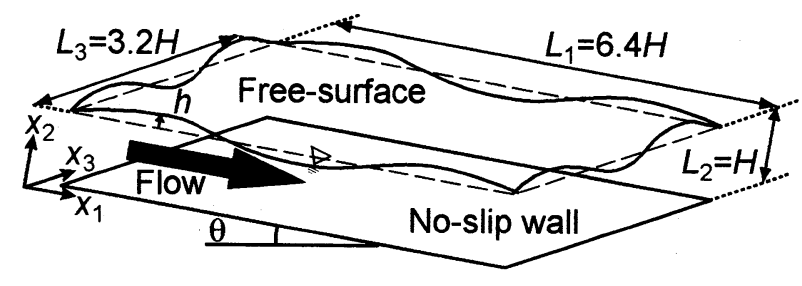

Fig.1 Configuration of open-channel flow with moving free surface.

\section{(2) Subgrid-scale stress model}

In order not to complicate the procedures and the method of interpretation, and to emphasize the effects of the free-surface fluctuation, we use the most-widely used Smagorinsky eddy-viscosity model. This allows direct comparison with the results of Kawamura ${ }^{2)}$ and Kawahara ${ }^{6)}$. The eddy viscosity model reads

$$
\begin{gathered}
\tau_{i j}=\frac{2}{3} k_{\mathrm{SGS}} \delta_{i j}-2 \nu_{\mathrm{SGS}}\left\langle S_{i j}\right\rangle, \\
k_{\mathrm{SGS}}=\frac{\nu_{\mathrm{SGS}}}{\left(C_{k} \Delta\right)^{2}}, \\
\nu_{\mathrm{SGS}}=\left(C_{s} \Delta\right)^{2} \sqrt{2\left\langle S_{i j}\right\rangle\left\langle S_{i j}\right\rangle} .
\end{gathered}
$$

We use the standard values recommended by Deardorff ${ }^{10)}$ for the model constants $C_{s}=0.1$, $C_{k}=0.094$ and $\Delta=\sqrt[3]{\Delta x_{1} \Delta x_{2} \Delta x_{3}}$. If these are substituted in Eq. (2), we obtain

$$
\begin{aligned}
\frac{D\left\langle u_{i}\right\rangle}{D t}= & -\frac{\partial}{\partial x_{i}}\left(\langle P\rangle+\frac{2}{3} k_{\mathrm{SGS}}\right)+g_{1} \delta_{1 i} \\
& +\frac{\partial}{\partial x_{j}}\left\{2\left(\nu+\nu_{\mathrm{SGS}}\right)\left\langle S_{i j}\right\rangle\right\} .
\end{aligned}
$$

\section{(3) Representation of the boundary condi-} tions on the free surface

In flows with subcritical Froude numbers, representation of the free surface is most conveniently done by using a continuous function defining the instantaneous position of the frec surfacc as explained earlier. If $\tilde{h}\left(x_{1}, x_{3}, t\right)$ is the instantaneous vertical position of the free surface at horizontal position $\left(x_{1}, x_{3}\right)$ and time $t$, the kinematic condition is

$$
\frac{\partial \tilde{h}}{\partial t}+\left.u_{\alpha}\right|_{\tilde{h}} \frac{\partial}{\partial x_{\alpha}} \tilde{h}=\left.u_{2}\right|_{\tilde{h}}
$$

where the repeated Greek subscript follows the usual summation convention but takes the horizontal directions 1 and 3 only, and $\left.()\right|_{\tilde{h}}$ means the quantity at $x_{2}=\tilde{h}=H+h$. The dynamic 
conditions are that the instantaneous normal and shear stresses on $x_{2}=\tilde{h}$ vanish.

We first apply the small-amplitude wave theory, which allows the above exact equations to be written in terms of the quantities at the mean position $x_{2}=H$. Then we perform the filtering operation to obtain the following equations for LES.

$$
\frac{\partial\langle h\rangle}{\partial t}+\frac{\partial}{\partial x_{\alpha}}\left(\left.\left\langle u_{\alpha}\right\rangle\right|_{H}\langle h\rangle\right)=\left.\left\langle u_{2}\right\rangle\right|_{H}-\frac{\partial \tau_{h \alpha}}{\partial x_{\alpha}}
$$

where

$$
\tau_{h \alpha}=\left\langle\left. h u_{\alpha}\right|_{H}\right\rangle-\left.\langle h\rangle\left\langle u_{\alpha}\right\rangle\right|_{H},
$$

and

$$
\begin{gathered}
\left.\left(\nu+\nu_{\mathrm{SGS}}\right)\left\langle S_{12}\right\rangle\right|_{H}=0, \\
\left.\left(\nu+\nu_{\mathrm{SGS}}\right)\left\langle S_{23}\right\rangle\right|_{H}=0, \\
\left.\langle p\rangle\right|_{H}=-g_{2}\langle h\rangle+\left.2\left(\nu+\nu_{\mathrm{SGS}}\right)\left\langle S_{22}\right\rangle\right|_{H} .
\end{gathered}
$$

\section{(4) Free-surface subgrid-scale model}

The new SGS term $\tau_{h \alpha}$ must now be modeled. As pointed out by the present authors ${ }^{9)}$, one method of modeling this term is to apply the gradient type modeling used for the SGS correlation between velocity and a passive scalar. Therefore, the first model is

$$
\tau_{h \alpha}=-C_{h 1} \nu_{\mathrm{SGS}} \frac{\partial\langle h\rangle}{\partial x_{\alpha}},
$$

where $C_{h 1}$ is a model constant and we use the value $C_{h 1}=5.0$ which is the value implied by the DNS data ${ }^{9)}$.

The second model is to apply the scalesimilarity model of Bardina et al. ${ }^{11)}$ According to this the SGS term $\tau_{h \alpha}$ is written as

$$
\tau_{h \alpha}=C_{B}\left(\left\langle\langle h\rangle\left\langle u_{\alpha}\right\rangle\right\rangle-\langle\langle h\rangle\rangle\left\langle\left\langle u_{\alpha}\right\rangle\right\rangle\right),
$$

where $C_{B}$ is a model constant, for which we use the value of $C_{B}=2.3$, again taken from the DNS data analysis. The scale-similarity model has been found to correlate the DNS data very well. It has also been known that it works well for the SGS stress as well, but it does not dissipate sufficient energy and is usually used with the dissipative eddy-viscosity model. In case of the present modeling of the free-surface fluctuation, it may be used by itself since there is no term corresponding to the viscous terms in the equation of the kinematic boundary condition.

\section{(5) Numerical methods and computational cases}

The numerical method of solution is the same as that used for the $\mathrm{DNS}^{8)}$ and is a finite difference technique based on the SMAC method on a Cartesian staggered grid. The spatial derivatives are discretized using the secondorder conservative difference scheme and time advancing is done by the second-order AdamsBashforth method. The position of the free surface $\langle h\rangle$ is solved by descretizing the spatial derivative terms in Eq. (11) using the fifth-order upstream-shifted interpolation(USI) scheme of Kajishima ${ }^{12)}$ and time advancing by the thirdorder Adams-Bashforth method. The Poisson equation for pressure is solved by Fouriertransforming the equations in the horizontal directions and the resulting equation is solved by the tri-diagonal algorithm.

The calculations were conducted for the Reynolds number $R e_{\tau}$ based on the friction velocity $u_{\tau}$ and the mean flow depth $H$ of 180 , and for the Froude number $F r$ based on the bulk average velocity and the mean depth $H$ of $0.3,0.6$ and 0.9 , which are the same as the present authors earlier DNS calculations ${ }^{8)}$. The number of grid points is $32^{3}$ compared with $128^{3}$ for the $\mathrm{DNS}^{8)}$. The ratio of the grid size of the LES calculation and that of the DNS is about 4 . We have found that the subgrid-scale contribution at this resolution is not very large but the effects should be recognizable.

With a time increment of $\Delta t^{+}=0.09$, about 200,000 time steps are used to obtain the wellconverged turbulence quantities averaged over space and time. The superscript ${ }^{+}$refers to the nondimensionalized quantities by $u_{\tau}$ and $\nu$.

In order to verify the entire numerical procedures, we have run the present computational code for an open-channel flow with rigid free-slip buundary at the mean free-surface position. The results of these calculations were then compared with the LES by Kawamura ${ }^{2)}$ and it was confirmed that the results were practically identical. Then nine simulations have been conducted altogether: three runs for three different Froude numbers using the two free-surface SGS models and without the free-surface SGS model at all.

\section{RESULTS AND DISCUSSION}

\section{(1) Mean velocity and Reynolds stress dis- tributions}

Figs. 2 through 4 show the distributions of the mean velocity, Reynolds shear stress and the turbulence intensities, for $F r=0.3,0.6$ and 0.9 , 


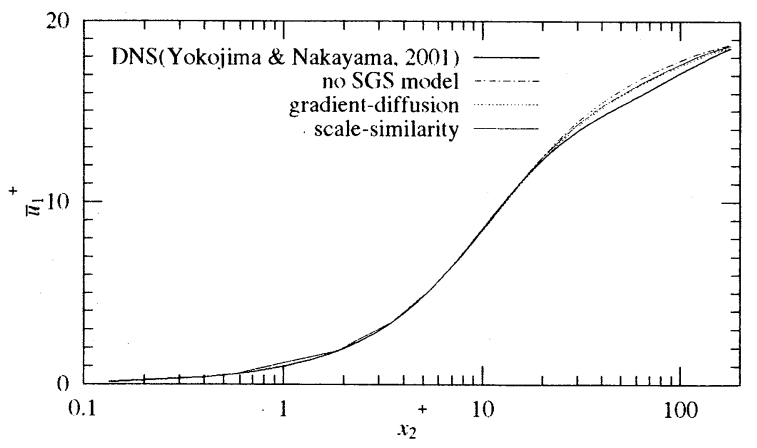

(a) Mean velocity

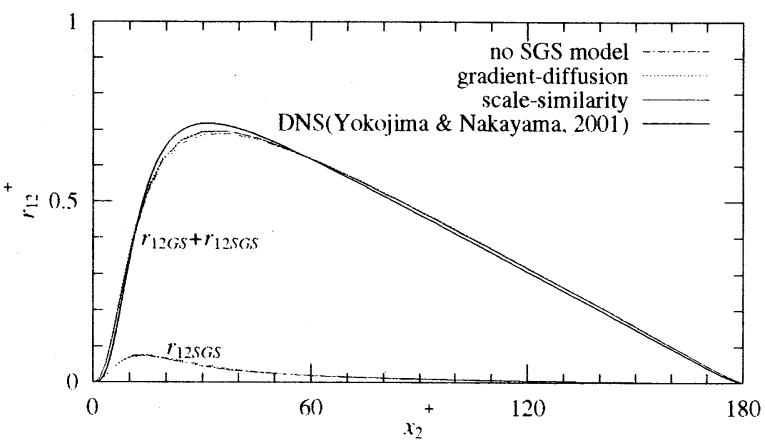

(b) Reynolds shear stress

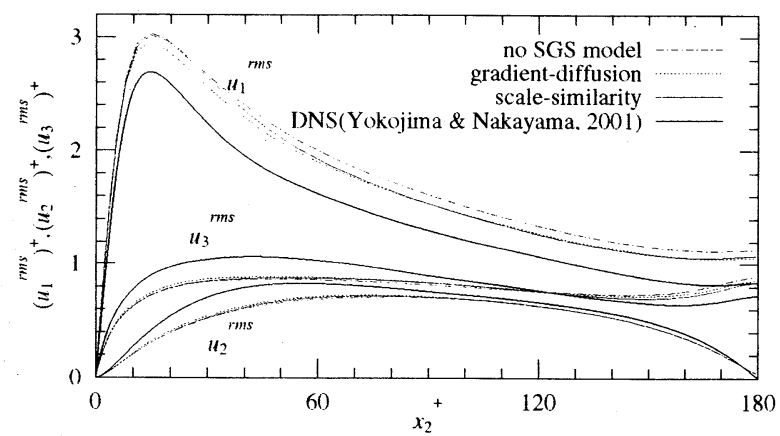

(c) Turbulence intensities

Fig.2 LES calculation results of open-channel flow for $F r=0.3$.

respectively, of the runs without the free-surface SGS terms and with the free-surface SGS terms modeled by the gradient diffusion and the scalesimilarity models. The corresponding $\mathrm{DNS}^{8)}$ results are also plotted for comparison. The mean velocity, Reynolds shear stress and the turbulence intensities normalized by $u_{\tau}$, are denoted by $\bar{u}_{1}^{\dagger}, r_{12}^{\dagger}$ and $\left(u_{i}^{\mathrm{rms}}\right)^{+}$, respectively. To be strict, the time averages of the LES results should be compared with the filtered time average of the DNS. Here the DNS mean velocity is the straight time average. In the plot of the shear stress, the contributions from the SGS stress $r_{12 \mathrm{SGS}} \equiv \overline{\tau_{12}}$ and the total of the contributions from the resolved motion $r_{12 \text { GS }} \equiv \overline{\left\langle u_{1}\right\rangle\left\langle u_{2}\right\rangle}-\overline{\left\langle u_{1}\right\rangle} \overline{\left\langle u_{2}\right\rangle}$ and the averages of the SGS stress are shown separately. In the actual calculation the time averages indicated by the overbar were evaluated by tak-

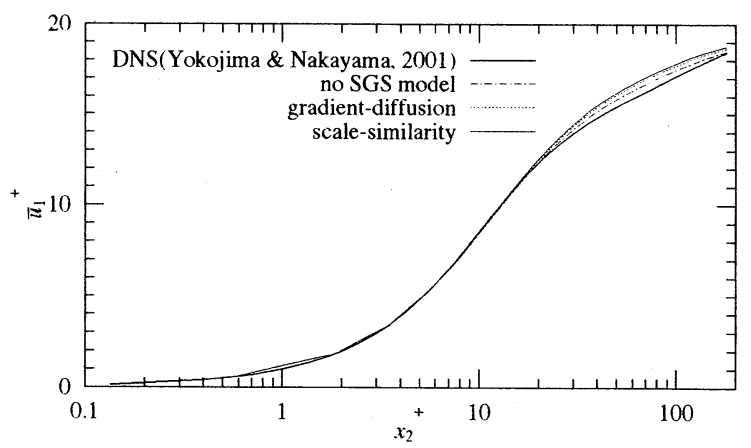

(a) Mean velocity

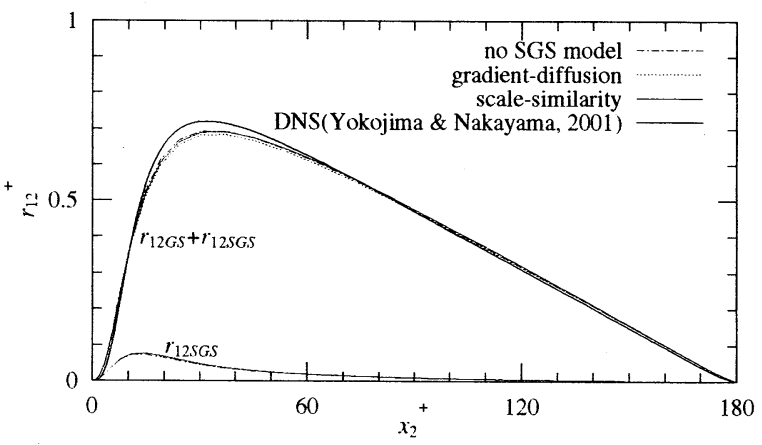

(b) Reynolds shear stress

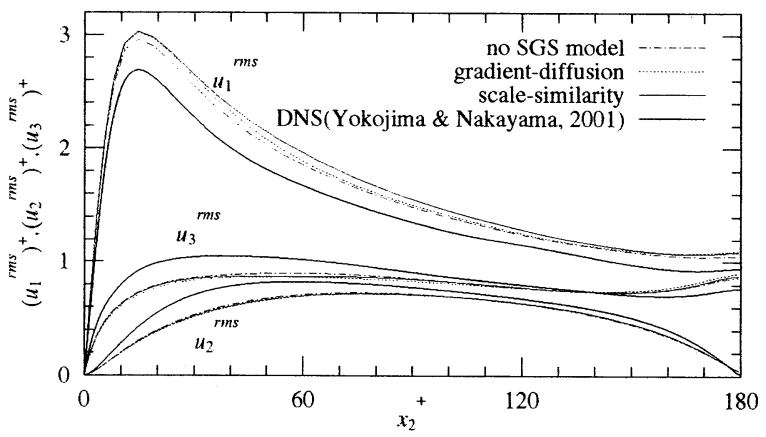

(c) Turbulence intensities

Fig.3 LES calculation results of open-channel flow for $F r=0.6$.

ing the average over time and over the horizontal plane. It is seen that for all models and for all Froude numbers for which calculations are made, the SGS contributions account for about 20 percent of the total stress at about $x_{2}^{+}=12$ where the SGS contribution is maximum, but they are less than 10 percent where the total Reynolds shear stress is maximum. The trends of the LES results of all these quantities are seen to be consistent with similar LES computations done for closed-channel flows (e.g. Morinishi et al. ${ }^{13)}$ ) and open-channel flows (Kawamura ${ }^{2)}$ ). In other words, the mean velocity is over-estimated in the logarithmic region, the streamwise intensity is over-estimated across the whole channel and the normal and transverse intensities are underestimated. 


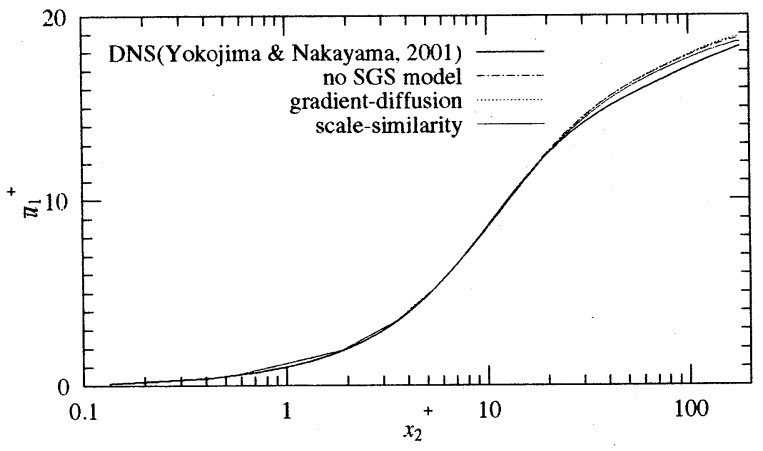

(a) Mean velocity

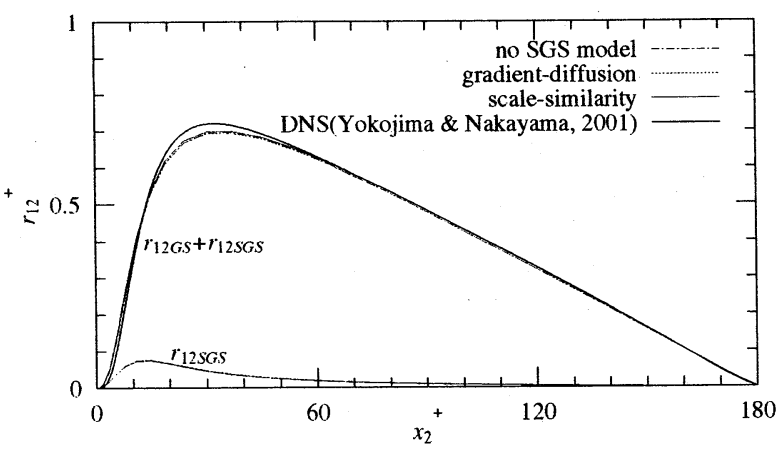

(b) Reynolds shear stress

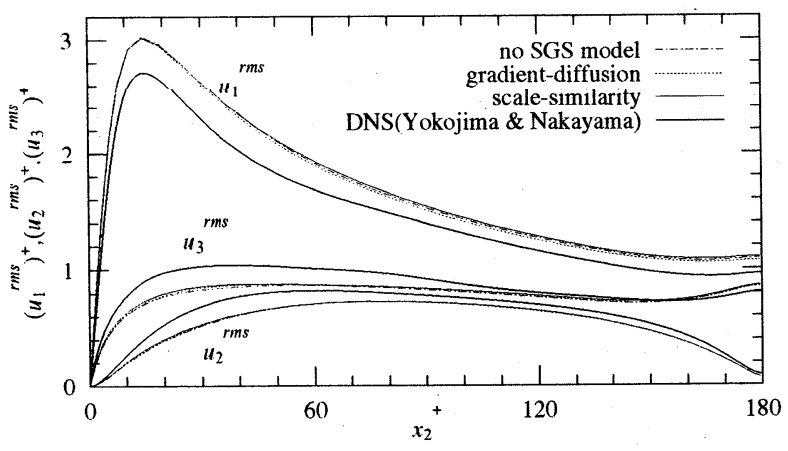

(c) Turbulence intensities

Fig.4 LES calculation results of open-channel flow for $F r=0.9$.

The differences among different models for the free-surface SGS terms are small, but the results without the free-surface SGS terms are seen to do poorest for the Froude number of 0.3 and 0.9 , indicating the importance of the filtering effects of the free surface. The scale-similarity model is seen to be slightly better than the gradient model. Since the gradient model is diffusive, it has an effect of attenuating the free-surface fluctuations. However, the governing equation for the free-surface elevation, which is the kinematic boundary condition, does not have a term corresponding to diffusion. Therefore, the model for the free-surface SGS is preferred to be one that does not have the diffusive nature. The scalesimilarity model may be a better choice for this reason, as well.

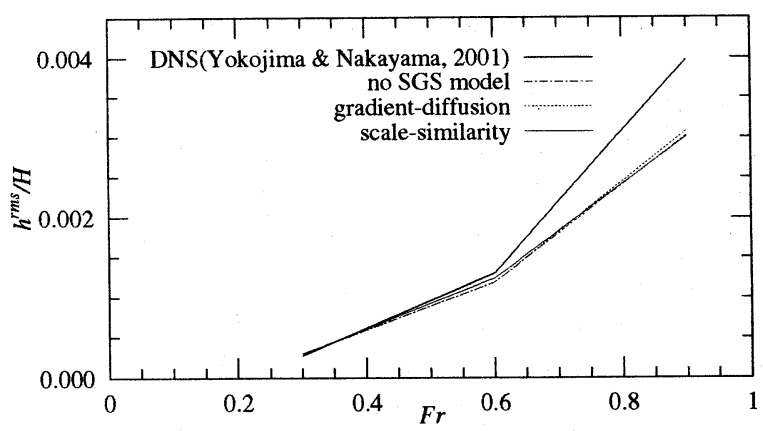

(a) RMS free-surface fluctuation

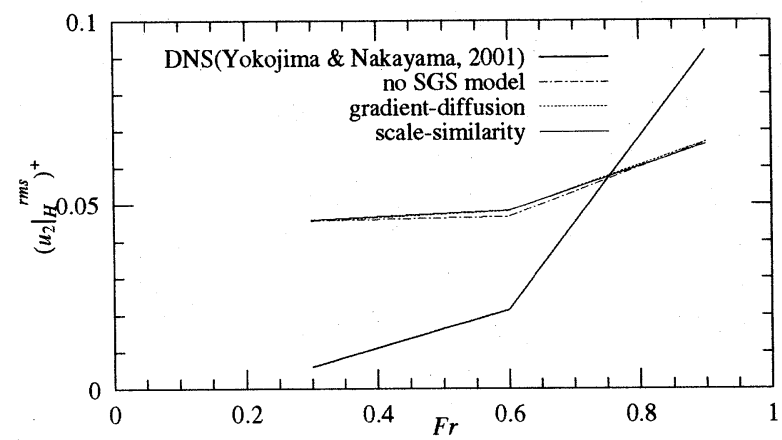

(b) Vertical turbulence intensity

Fig.5 RMS free-surface fluctuation and vertical intensity on the free surface.

(2) Free surface fluctuation and vertical velocity fluctuation at the free surface

In order to see the effects on the motion of the free surface more directly, the results of the RMS amplitudes of the free-surface fluctuation $h^{r m s}$ and the vertical velocity fluctuation on the free surface are plotted in Fig. 5 and compared with the $\mathrm{DNS}^{9)}$. The difference among all LES calculations are small, but definite difference is seen between the LES and $\mathrm{DNS}^{9)}$ results. The difference in $h^{r m s}$ is considered to be due to the free-surface SGS contribution, $\tau_{h h} \equiv\left\langle h^{2}\right\rangle-\langle h\rangle^{2}$, which is not included in this plot. The results suggest a model

$$
\tau_{h h}=\left(C_{h 2} \Delta\right)^{2} \sqrt{\frac{\partial\langle h\rangle}{\partial x_{\alpha}} \frac{\partial\langle h\rangle}{\partial x_{\alpha}}}
$$

with the value of the constant $C_{h 2}$ of the order of 0.1 , is a good representation. The DNS results ${ }^{9)}$ indicated that the length scale of the free-surface fluctuation is larger than the viscous scales. Since nearly 80 percent of the total stress near the bottom is resolved, it is expected much better percentage of the free surface fluctuation is resolved. That is the main reason for small differences among different models. 


\section{CONCLUSIONS}

Large-eddy simulation of fully-developed turbulent open-channel flow has been conducted allowing motion of the free surface. The shape of the free surface, and hence flow domain, is filtered along with the flow field itself. Filtering of the free surface position introduces extra SGS terms. Two models for these SGS terms have been formulated and incorporated in the calculations. Their importance has been examined by conducting calculations with these SGS terms and without them. The results indicate that the effects of these terms may not be overwhelmingly important in subcritical flows at low Reynolds number examined here, but some differences are seen in the mean-velocity profile near the free surface and in the turbulent stresses. Their role in more practical case at higher Reynolds number and Froude number and in spatially varying flows can be important and should be examined.

\section{REFERENCES}

1) Thomas, T.G., Williams, J.J.R. and Leslie, D.C.: Development of a conservative 3D free surface code, $J$. Hydraulic Research, Vol.30 No.1, pp.107-115, 1992.

2) Kawamura, T.: Numerical simulation of $3 \mathrm{D}$ turbulent free-surface flows, Ph.D. Thesis, International Research Centre for Computational Hydrodynamics, Horsholm, Denmark, 1998.

3) Hodges, B.R. and Street, R.L.: On simulation of turbulence nonlinear free-surface flows, J. Comput. Phys., Vol.151, pp.425-457, 1999.
4) Dimas, A.A. and Fialkowski, L.T.: Large-wave simulation(LWS) of free-surface flows developing weak splilling breaking waves, J. Comput. Phys., Vol.159, pp.172-196, 2000.

5) Shen, L. and Yue, D.K.P.: Large-eddy simulation of free-surface turbulence, J. Fluid Mech., Vol.440, pp.75116, 2001.

6) Kawahara, Y.: Modeling of free surface effects on turbulent flow in open channel, Proc. 2001 Meeting of $J S F M$, pp.59-60, 2001(in Japanese).

7) Onitsuka, K. and Nezu, I.: Numerical prediction of rectangular open-channel flow by using large eddy simulation, 29th IAHR Congress Proc., Theme D, Vol.1, pp.196-201, 2001.

8) Yokojima, S. and Nakayama, A.: DNS study of Froude number effects on turbulence structure near free surface, J. Applied Mech. JSCE, Vol.4, pp.619-628, 2001(in Japanese).

9) Yokojima, S. and Nakayama, A.: Filtering effects of free-surface fluctuations in LES of open-channel turbulent flows, submitted to Annual J. Hydraulic Eng. $J S C E$, Vol.46(in Japanese).

10) Deardorff, J.W.: A numerical study of threedimensional turbulent channel flow at large Reynolds numbers, J. Fluid Mech., Vol.41, pp.453-480, 1970.

11) Bardina, J., Ferziger, J. and Reynolds, W.C.: Improved turbulence models based on large-eddy simulation, AIAA Paper, 80-1357, 1980.

12) Kajishima, T.: Upstream-shifted interpolation method for numerical simulation of incompressible flows, Trans. JSME B, Vol.60 No.578, pp.3319-3326, 1994(in Japanese).

13) Morinishi, Y., Ichikawa, A., Okumura, T. and Nakabayashi, K.: Effects of the order or accuracy of finite-difference method and grid resolution on LES of wall turbulence, Trans. JSME B, Vol.66 No.647, pp.1750-1757, 2000(in Japanese).

(Received October 1, 2001) 